\title{
Surface Energies of Simple Metals from Slabs: Comparison of Exchange- Correlation Density Functionals
}

\author{
CARLOS FIOLHAIS, ${ }^{1,2}$ L. M. ALMEIDA ${ }^{2,3}$ \\ ${ }^{1}$ Department of Physics, University of Coimbra, 3004-516 Coimbra, Portugal \\ ${ }^{2}$ Center for Computational Physics, University of Coimbra, 3004-516 Coimbra, Portugal \\ ${ }^{3}$ Department of Physics, University of Aveiro, 3810 Aveiro, Portugal
}

Received 30 September 2003; revised 27 November 2003; accepted 8 December 2003

Published online 3 November 2004 in Wiley InterScience (www.interscience.wiley.com).

DOI 10.1002/qua.20321

\begin{abstract}
Surface energies of some simple metals (Be, $\mathrm{Al}, \mathrm{Mg}, \mathrm{Li}$, and $\mathrm{Na}$ ) were calculated within density functional theory. Various approximations for the exchange and correlation were tested: local density approximation (LDA), generalized gradient approximations (GGA), and meta-generalized gradient approximation (MGGA). We used the Crystal98 code to calculate slabs with one to 10 layers using both all-electron and pseudopotential approaches. The extraction of the surface energy from the series of slab energies was done by the linear-fitting method. Our LDA and GGA results agree reasonably well with those from other authors. The MGGA values are, in general, close to LDA and higher than GGA ones, a pattern also observed in the jellium model. They also agree reasonably well with the experimental data. $\quad$ (C) 2004 Wiley Periodicals, Inc. Int J Quantum Chem 101: 645-650, 2005
\end{abstract}

Key words: density functional theory; Gaussian-type orbitals; meta generalized gradient approximation; simple metals; slabs; surface energies

\section{Introduction}

$\mathbf{T}$ he importance of the surface energy is well known in surface science and technology. It plays an essential role in understanding physical

Correspondence to: C. Fiolhais; e-mail: tcarlos@teor.fis.uc.pt Contract grant sponsor: Fundação para a Ciência e Tecnologia, Portugal.

Contract grant number: POITI/CM/41574/2001. processes such as adhesion and corrosion. It represents also the main contribution to the vacancy formation energy [1]. Surface energies are measured only indirectly. The difficulty in obtaining good experimental values reinforces the need for accurate calculations.

The extraction of the surface energy of a given material from a series of slabs with different thickness turns out to be easier than the calculation of the semi-infinite system. But then the extrapolation from thin slabs to the semi-infinite system is neces- 
sary. The main drawback of thin-slab calculations is the occurrence of quantum-size effects, that is, energy fluctuations due to the finite size of the slab [2].

The energy of a slab with $N$ layers may be written as the sum of a bulk term, which is proportional to the volume $V$, and a surface term, which is proportional to the area $S$ :

$$
E_{\text {slab }}=\epsilon_{\text {bulk }} V+2 \sigma S \text {, }
$$

where $\epsilon_{\text {bulk }}$ is the energy density of the bulk crystal and $\sigma$ is the surface energy. From this equation, we may extract the surface energy

$$
\sigma=\frac{1}{2 S}\left(E_{\text {slab }}-\epsilon_{\text {bulk }} V\right) .
$$

The surface energy is expected to be very sensitive to the two parcels from which it is calculated, because it is small compared with them.

Boettger [3] pointed out a technical problem associated with Eq. (1.2): its right side diverges linearly with the number of layers in the slab. Because of the separate calculation of $\epsilon_{\text {bulk }}$, the bulk contribution to the slab energy is not canceled by the energy of a bulk piece with the same size. Various methods for evaluating the surface energy from slab energies that avoid an independent calculation of the bulk energy have been proposed [3-6].

Here we present one of these methods. Rearranging Eq. (1.2) we obtain

$$
\frac{E_{\text {slab }}(N)}{S}=d N \epsilon_{\text {bulk }}+2 \sigma,
$$

where $d$ is the slab thickness and $N$ the number of layers. Using a series of slabs with different $N$, it is possible to fit the parameters $\epsilon_{\text {bulk }}$ and $\sigma$. This linear fitting method [4] is more reliable than the incremental method, which requires only two slabs with consecutive number of layers [7].

The slab energies $E_{\text {slab }}$ may be obtained solving the Kohn-Sham equations of density functional theory [8]. The generalized gradient approximation (GGA), whose inputs are the density and its gradient, goes beyond the original local density approximation (LDA) for exchange and correlation. A widely used GGA is Perdew-Burke-Ernzerhof (PBE) [9]. Another GGA was proposed by Becke [10] for exchange, which, together with the LeeYang-Parr [11] correlation, results in a functional widely used by chemists, known as BLYP. The PBE is constructed in a nonempirical way, whereas BLYP is semiempirical, that is, it contains parameters adjusted to molecular data. A step beyond GGA in the hierarchy of functionals towards the "exact" one is the meta-generalized gradient approximation (meta-GGA, or MGGA), a scheme that includes the kinetic energy density in the exchange and correlation functional together with the density and its gradient. One of the most widely used MGGA functionals is PKZB [12], which was designed by Perdew and co-workers (Kurth, Zupan, and Blaha) to respect more exact constraints than PBE and which contains a single parameter adjusted to experimental data. An improvement of PKZB that is completely free from parameters adjusted to experimental data was proposed recently [13], but its results should not differ much from PKZB ones.

In this work we present surface energies of a set of simple metals based on different density functionals. For LDA we adopted the Perdew-Wang 92 [14] parametrization of the correlation energy, which is based on the release-node diffusion Monte Carlo calculation of Ceperley and Alder [15] for the uniform electron gas. Beyond LDA the energies were calculated with PBE densities. It has been shown [16-18] that the energy corrections due to full self-consistency are typically small.

All surface energies are extracted from slab energies with unrelaxed ionic planes (faces (111), (110), and (100) for fcc and bcc structures and (0001) face for hcp structures). The relaxation of the outermost layers is experimentally known and confirmed by ab initio calculations [19]. However, the influence of this effect on the surface energy is quite small [19-21] (typically 2-5\%). We do not aim with this work to obtain optimal energies but to compare different exchange-correlation functionals. We believe that the differences between surface energies given by the various functionals will remain close to the values found here after structure optimization.

After describing the computational method in the second section, our results are shown and discussed in the third section. Short conclusions appear at the end.

\section{Computational Method}

All calculations presented in this work were performed with the Crystal98 program package [22]. 
They are either all-electron (full potential) or pseudopotential (effective core potential), always using Gaussian basis sets. Pseudopotentials offer the advantage of dealing with few electrons and having a much reduced energy scale. The chosen pseudopotential was the Durand-Barthelat [23], because it is implemented in the code.

The PBE and BLYP functionals for exchange and correlation are options in Crystal98. However, the PKZB functional is not part of the standard code and had to be inserted by us [17].

To obtain surface energies we performed a series of slab calculations, going from one to 10 atomic layers. In all-electron calculations, the exponents of the uncontracted Gaussians of the most outer shells belonging to the basis sets referred to in Ref. [17] were reoptimized for slabs with three layers. For sodium a $d$ shell was added to the basis sets in order to improve the surface energy. The basis sets used in pseudopotential calculations are shown in Table I. The exponents were optimized as in the all-electron case.

The convergence of the energy with respect to the number of special $\mathbf{k}$ points within the MonkhorstPack [24] scheme was checked. A shrinking factor of 16 in the Monkhorst-Pack net was found to be adequate for all our slabs. A temperature smearing of 0.001 hartree in the Fermi surface was also used, to improve the iteration procedure.

To extract the surface energy we have adopted the fitting procedure expressed by Eq. (1.3) and explained by Fiorentini and Methfessel [4]. The quantum-size effects exhibited by ultrathin slabs is the most severe problem in extracting the surface energy. The confirmed expectation is that they become smaller for larger $N$ [7]. Figure 1 illustrates the influence of the number of slabs taken in the fit. Our final results, shown in the third section, were obtained, considering always the whole set of 10 slabs.

\section{Results}

Table II shows the surface energies we have found for the various density functionals. The LDA

\section{TABLE I}

Basis sets used for the Durand-Barthelat pseudopotential calculations.

\begin{tabular}{lccccc}
\hline Metal & $\mathrm{Be}$ & $\mathrm{Al}$ & $\mathrm{Mg}$ & $\mathrm{Li}$ & $\mathrm{Na}$ \\
\hline Basis set & $111 \mathrm{G}$ & $11(\mathrm{~d} 1) \mathrm{G}$ & $11(\mathrm{~d} 1) \mathrm{G}$ & $11(\mathrm{~d} 1) \mathrm{G}$ & $111(\mathrm{~d} 1) \mathrm{G}$
\end{tabular}

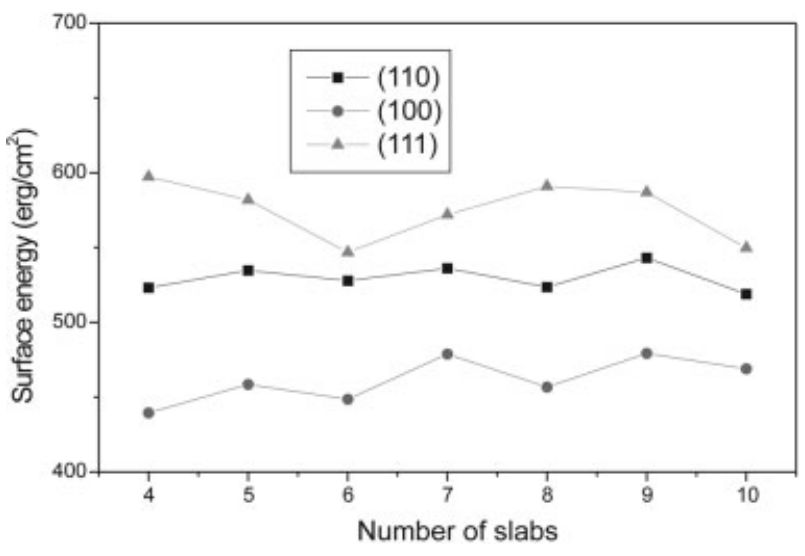

FIGURE 1. Dependence of the PBE surface energy of lithium on the number of fitted slabs (all-electron results). The slabs used in the fitting are always the thickest ones up to 10 layers.

and PBE values resulted from fully self-consistent calculations, whereas PKZB and BLYP used the PBE density. The lattice constants were chosen to take the PBE values used by Vitos et al. [25], who applied the full-charge density method, derived from the linear muffin-tin orbital method, for the semi-infinite system. Given the uncertainty associated with the fitting method, our all-electron PBE results are in good agreement with those of Vitos et al. [25].

Our pseudopotential values are, in general, close to all-electron ones. The current LDA pseudopotential calculations agree in general with reported ones [21, 28, 32-34]. We note that our LDA values are all higher than PBE ones, in agreement with the jellium model prediction [30] and with real metals results [25]. The fact that LDA values reported by various authors (see Table II) are lower than the PBE values of Ref. [25] is due to the use of different methods and approximations in those calculations.

For both all-electron and pseudopotentials, PKZB surface energies lie between the LDA and PBE ones, with the exception of aluminum.

Regarding the face anisotropy, we obtain for PBE the same trend as in Ref. [25], with the exception of aluminum. Using LDA and pseudopotentials the agreement with the anisotropy found by other authors is complete.

Our results agree with experimental data. However, the comparison between calculated and experimental surface energies has to be done with care. Experimental values, which are obtained for an undifferentiated face, are not very reliable. They are 
TABLE II

Surface energies of simple metals obtained from fitting the Crystal98 energies of a series of slabs with 1 to 10 layers. $^{a}$

\begin{tabular}{|c|c|c|c|c|c|c|c|c|c|}
\hline \multirow[b]{2}{*}{ Metal } & \multirow{2}{*}{$\begin{array}{l}\text { Structure and } \\
\text { lattice constant }\end{array}$} & \multirow[b]{2}{*}{ Surface } & \multicolumn{4}{|c|}{ Current work } & \multicolumn{3}{|c|}{ Other works } \\
\hline & & & LDA & PBE & PKZB & BLYP & LDA & $\mathrm{PBE}^{\mathrm{b}}$ & Experiment \\
\hline \multirow[t]{2}{*}{$\mathrm{Be}$} & hcp & (0001) & 2273 & 1677 & 1766 & 1110 & & 1834 & $1628,^{c} 2700^{d}$ \\
\hline & 2.236 & & 2180 & 1865 & 2082 & 1273 & $1924,^{e} 2100^{f}$ & & \\
\hline \multirow[t]{6}{*}{$\mathrm{Al}$} & fcc & (111) & 1272 & 1103 & 1280 & 636 & & 1199 & $1143,{ }^{b} 1160^{c}$ \\
\hline & & & 1196 & 1002 & 1097 & 570 & $1016,^{g} 939^{h}$ & & \\
\hline & 4.049 & (100) & 1499 & 1326 & 1527 & 820 & & 1347 & \\
\hline & & & 1411 & 1220 & 1309 & 772 & $1081^{i}$ & & \\
\hline & & (110) & 1543 & 1358 & 1551 & 829 & & 1271 & \\
\hline & & & 1436 & 1229 & 1312 & 758 & $1090^{\mathrm{j}}$ & & \\
\hline \multirow[t]{2}{*}{$\mathrm{Mg}$} & hcp & (0001) & 777 & 682 & 745 & 427 & & 792 & $785,^{c} 760^{d}$ \\
\hline & 3.196 & & 677 & 584 & 624 & 335 & $641^{\mathrm{k}}$ & & \\
\hline \multirow[t]{6}{*}{$\mathrm{Li}$} & bcc & (110) & 608 & 519 & 553 & 367 & & 556 & $522,^{c} 525^{d}$ \\
\hline & & & 569 & 516 & 543 & 362 & $545^{1}$ & & \\
\hline & 3.431 & (100) & 565 & 469 & 489 & 338 & & 522 & \\
\hline & & & 511 & 468 & 488 & 332 & $506^{\prime}$ & & \\
\hline & & (111) & 634 & 550 & 564 & 431 & & 590 & \\
\hline & & & 618 & 578 & 592 & 453 & $623^{1}$ & & \\
\hline \multirow[t]{6}{*}{$\mathrm{Na}$} & bcc & (110) & 297 & 229 & 260 & 127 & & 253 & $261,{ }^{c} 260^{d}$ \\
\hline & & & 250 & 223 & 228 & 126 & & & \\
\hline & 4.197 & (100) & 323 & 242 & 267 & 144 & & 264 & \\
\hline & & & 254 & 235 & 240 & 147 & & & \\
\hline & & (111) & 359 & 294 & 329 & 187 & & 287 & \\
\hline & & & 304 & 279 & 278 & 189 & & & \\
\hline
\end{tabular}

a The first row for each face shows all-electron results, whereas the second row shows pseudopotential results. Lattice constants are in $\AA$. The ideal ratio $c / a=2 / 3 \sqrt{6}$ was adopted for hcp structures. All energies are in $\mathrm{erg} / \mathrm{cm}^{2}$.

${ }^{\mathrm{b}}$ Ref. [25].

${ }^{\mathrm{c}}$ Ref. [26].

${ }^{\mathrm{d}}$ Ref. [31].

e Ref. [21].

${ }^{f}$ Ref. [32].

${ }^{g}$ Ref. [2].

${ }^{\mathrm{h}}$ Ref. [28].

'Ref. [29].

${ }^{\text {i }}$ Ref. [20].

${ }^{\mathrm{k}}$ Ref. [33].

'Ref. [34].

obtained from measurements of the gas-solid surface tension, which has to be extrapolated to zero temperature [26]. Another way to obtain surface energies uses elastic constants and bulk modulus measurements [27]. Thus, we give more importance to the comparison of our values with good ab initio results than to comparison with experimental data.

The BLYP surface energies are always too low compared with those of the other functionals and with experimental data so that this functional does not seem to be adequate for metal surfaces.

In Table III we present pseudopotential exchange-correlation surface energies. A straightfor- ward comparison can now be made with the jellium model and, to facilitate that comparison, all surface energies are now based on LDA densities. In the jellium model, which has proved very useful to describe some properties of simple metals, the ionic structure of solids is replaced by a uniform positive-charge density. For each metal, jellium values are indicated in Table III. We see that PKZB values are close but above LDA ones. On the other hand, for real metals, PKZB results are also close to LDA, although not necessarily above them. This result is a bit surprising. A formula that reproduces the jellium exchange-correlation surface energy as a 
function of the valence-electron density parameter $r_{s}$ can be found in Ref. [18]. Figure 2 compares the LDA exchange-correlation surface energies of the jellium model given by that formula with real-metal results. The conclusion is that the trend given by jellium model is indeed followed by real systems. The same holds for the other functionals.

We note that, in the jellium model, PBE underestimates what is known to be the most accurate exchange-correlation surface energy for that model [30], whereas LDA and PKZB are close to that "exact" value. It is astonishing that LDA works so well in such a strongly inhomogeneous situation, but this is certainly due to a cancellation of exchange and correlation errors.

\section{Conclusions}

We used Crystal98 to calculate slabs of simple metals with up to 10 layers to extract surface energies. The results found for $\mathrm{Be}, \mathrm{Al}, \mathrm{Mg}, \mathrm{Li}$, and $\mathrm{Na}$ agree well with the results of other authors and with experimental data, so that the use of localized Gaussian functions as basis sets seems appropriate even for that class of metals, in which the valence

TABLE III

\begin{tabular}{|c|c|c|c|c|c|c|}
\hline & $r_{s}$ (bohr) & Surface & LDA & PBE & PKZB & BLYP \\
\hline \multirow[t]{2}{*}{$\mathrm{Be}$} & 1.85 & jellium & 4448 & 4334 & 4511 & 3730 \\
\hline & & (0001) & 3622 & 3467 & 3681 & 2879 \\
\hline \multirow[t]{4}{*}{$\mathrm{Al}$} & 2.07 & jellium & 2961 & 2880 & 3003 & 2424 \\
\hline & & (111) & 2558 & 2467 & 2560 & 2039 \\
\hline & & (100) & 2884 & 2795 & 2883 & 2353 \\
\hline & & (110) & 2973 & 2875 & 2958 & 2410 \\
\hline \multirow[t]{2}{*}{ Mg } & 2.65 & jellium & 1204 & 1167 & 1221 & 916 \\
\hline & & (0001) & 976 & 930 & 970 & 681 \\
\hline \multirow[t]{4}{*}{$\mathrm{Li}$} & 3.19 & jellium & 609 & 589 & 618 & 427 \\
\hline & & (110) & 521 & 495 & 522 & 340 \\
\hline & & (100) & 629 & 607 & 628 & 471 \\
\hline & & (111) & 643 & 623 & 638 & 498 \\
\hline \multirow[t]{4}{*}{$\mathrm{Na}$} & 3.91 & jellium & 280 & 270 & 284 & 171 \\
\hline & & (110) & 239 & 226 & 232 & 129 \\
\hline & & (100) & 315 & 305 & 310 & 217 \\
\hline & & (111) & 307 & 295 & 294 & 205 \\
\hline
\end{tabular}

a The LDA densities are used overall. The second column shows the bulk valence-electron densities for which the jellium values were calculated. Energies are in $\mathrm{erg} / \mathrm{cm}^{2}$.

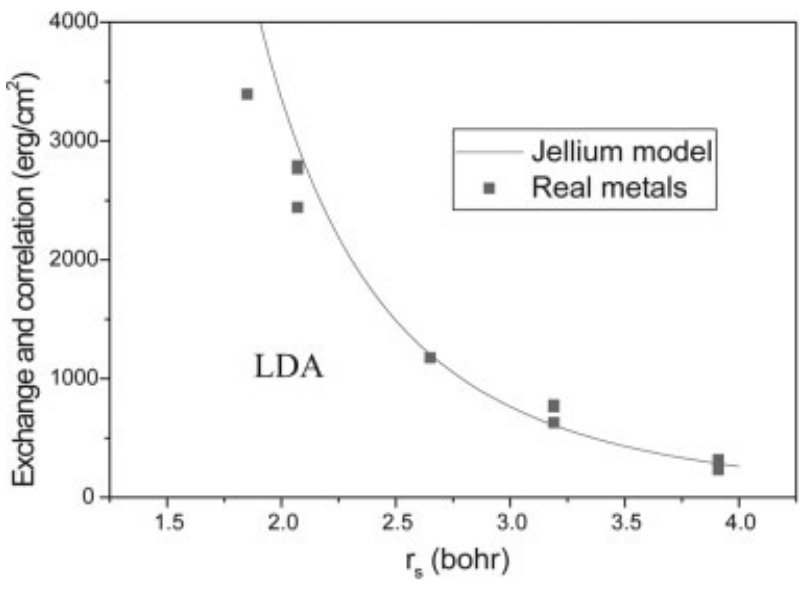

FIGURE 2. Comparison of realistic LDA exchangecorrelation surface energies with the prediction of the jellium model. The squares represent the exchangecorrelation surface energies of real metals (different points for the same density refer to different exposed faces), whereas the solid line represents the same component of the surface energy using Eq. (15) of Ref. [18].

electrons are nearly free. Our LDA surface energies of real metals are higher than PBE ones, as in the jellium model. The PKZB surface energies are between the LDA and PBE energies, except for some aluminum cases. The available experimental data cannot decide which one of the three functionals is best for metal surfaces but can exclude BLYP, which is popular for molecules.

\section{ACKNOWLEDGMENTS}

This work was in part supported by the project "Computational Nanophysics" (POITI/CM/41574/ 2001) granted by Fundação para a Ciência e Tecnologia, Portugal.

\section{References}

1. Perdew, J. P.; Wang, Y.; Engel, E. Phys Rev Lett 1991, 66, 508.

2. Boettger, J. C. Phys Rev B 1996, 53, 13133.

3. Boettger, J. C. Phys Rev B 1994, 49, 16798.

4. Fiorentini, V.; Methfessel, M. J Phys Condensed Matter 1996, 8,6525 .

5. Boettger, J. C.; Smith, J. R.; Birkenheuer, U.; Rösch, N.; Trickey, S. B.; Sabin, J. R.; Apell, S. P. J Phys Condensed Matter 1998, 10, 893.

6. Fiorentini, V.; Methfessel, M. J Phys Condensed Matter 1998, 10, 895. 
7. Fiolhais, C.; Almeida, L. M.; Henriques, C. Prog Surf Sci 2003, 74, 209.

8. Kohn, W.; Sham, L. J. Phys Rev A 1965, 140, 1133.

9. Perdew, J. P.; Burke, K.; Ernzerhof, M. Phys Rev Lett 1996, 77, 3865.

10. Becke, A. D. Phys Rev A 1988, 38, 3098.

11. Lee, C.; Yang, W.; Parr, R. G. Phys Rev B 1988, 37, 785.

12. Perdew, J. P.; Kurth, S.; Zupan, A.; Blaha, P. Phys Rev Lett 1999, 82, 2544.

13. Tao, J.; Perdew, J. P.; Staroverov, V. N.; Scuseria, G. E. cond-mat/0306203.

14. Perdew, J. P.; Wang, Y. Phys Rev B 1992, 45, 13244.

15. Ceperley, D. M.; Alder, B. J. Phys Rev Lett 1980, 45, 566.

16. Fan, L.; Ziegler, T. J Chem Phys 1991, 94, 6057.

17. Almeida, L. M.; Fiolhais, C.; Causà, M. Int J Quantum Chem 2003, 91, 224.

18. Almeida, L. M.; Perdew, J. P.; Fiolhais, C. Phys Rev B 2002, 66, 075115.

19. Holzwarth, N. A. W.; Zeng, Y. Phys Rev B 1995, 51, 13653.

20. Ho, K. M.; Bohnen, K. P. Phys Rev B 1985, 32, 3446.

21. Feibelman, P. J. Phys Rev B 1992, 46, 2532.
22. Saunders, V. R.; Dovesi, R.; Roetti, C.; Caus, M.; Harrison, N. M.; Orlando, R.; Zicovich-Wilson, C. M. Crystal98 User's Manual; University of Torino: Torino, 1998.

23. Durand, P.; Barthelat, J. C. Theor Chim Acta 1975, 38, 283.

24. Monkhorst, H. J.; Pack, J. D. Phys Rev B 1976, 13, 5188.

25. Vitos, L.; Ruban, A. V.; Skriver, H. L.; Kollár, J. Surf Sci 1998, 411, 186.

26. Tyson, W. R.; Miller, W. A. Surf Sci 1977, 62, 267.

27. Wawra, H. Z Metallkd 1975, 66, 395.

28. Schöchlin, J.; Bohnen, K. P.; Ho, K. M. Surf Sci 1995, 324, 113.

29. Bohnen, K. P.; Ho, K. M. Surf Sci 1995, 207, 105.

30. Kurth, S.; Perdew, J. P.; Blaha, P. Int J Quantum Chem 1999, 75,889 .

31. de Boer, F. R.; Boom, R.; Mattens, W. C. M.; Miedema, A. R.; Niessen, A. K. Cohesion in Metals; North-Holland: Amsterdam, 1988.

32. Yu, R.; Lam, P. K. Phys Rev B 1989, 39, 5035.

33. Wright, A. F.; Feibelman, P. J.; Atlas, S. R. Surf Sci 1994, 302, 215.

34. Kokko, K.; Salo, P. T.; Laihia, R.; Mansikka, K. Surf Sci 1994, $348,168$. 